\title{
Thapsigargin Induces Platelet Aggregation, thereby Releases Lactate Dehydrogenase from Rat Platelets
}

\author{
Ji Sue Baik ${ }^{1,3, \S, *}$, You Na Seo ${ }^{1,, *}$, Man Hee Rhee ${ }^{2, * * *}$, Moon-Taek Park ${ }^{1, \dagger, * *}$ \\ and Sung Dae Kim ${ }^{2, \dagger ; * * *}$ \\ ${ }^{I}$ Research Center, Dongnam Institute of Radiological \& Medical Sciences, Busan 46033, Korea \\ ${ }^{2}$ Department of Veterinary Medicine, College of Veterinary Medicine, \\ Kyungpook National University, Daegu 41566, Korea \\ ${ }^{3}$ Department of Medicinal Biotechnology, College of Health Sciences, \\ Dong-A University, Busan 49315, Korea
}

\begin{abstract}
Thapsigargin (TG), a sarco/endoplasmic reticulum (ER) $\mathrm{Ca}^{2+}$-ATPase (SERCA) inhibitor, has been widely used as an agonist for platelet aggregation for decades. In this study, we investigated the effect of TG on the release of lactate dehydrogenase $(\mathrm{LDH})$ for platelets and elucidated its mechanism. Platelet LDH release and platelet aggregation were increased by TG treatment; 1,000 nM of TG induced the complete lysis of platelets. Other agonists such as collagen $(2.5 \mu \mathrm{g} / \mathrm{mL})$, thrombin $(0.1 \mathrm{U} / \mathrm{mL})$, and ADP $(10 \mathrm{mM})$ did not induce significant platelet LDH release despite platelet aggregation. Finally, we investigated the effects of pharmacological inhibitors on TG-induced platelet aggregation and LDH release. SP600125, a JNK inhibitor, and LY294002, a PI-3K inhibitor, inhibited TG-induced platelet LDH release but not platelet aggregation. Forskolin, an adenylyl cyclase activator, also inhibited LDH release without affecting platelet aggregation by TG. These results suggest that the TG-induced platelet aggregation was accompanied by LDH release but regulated by a different signaling pathway.
\end{abstract}

Key Words: Platelets, Thapsigargin, Lactate dehydrogenase, cAMP, JNK, PI-3K

Platelets are non-nucleated cells that are formed by detaching from megakaryocyte cells. Resting-state platelets circulate in the blood for 7 to 10 days. Platelets are activated by a variety of agonists such as collagen, ADP, arachidonic acid, and epinephrine, resulting in altered structure, aggregation, and the formation of a blood clot. Platelet aggregation is a normal response of the body to various pathophysi- ological phenomena. Lactate dehydrogenase (LDH) is a hydrogen-transfer enzyme that is found in the cytoplasm of most cells. The secretion of LDH is widely used to assess cell viability and late-stage apoptosis. Platelets carry all the LDH isoenzymes including LDH-1 to LDH-5 (Patton et al., 1994). Therefore, platelet LDH has been measured to determine anti-platelet drug toxicity. Exposure to TG resulted in

Received: September 1, 2021 / Revised: September 17, 2021 / Accepted: September 17, 2021

${ }^{*}$ Researcher, ${ }^{* *}$ Senior Researcher, ${ }^{* * *}$ Professor.



${ }^{\dagger}$ Corresponding author: Moon-Taek Park. Research Center, Dongnam Institute of Radiological \& Medical Sciences, Busan, Korea. Tel: +82-51-720-5141, Fax:+82-51-720-5929, e-mail: mtpark@dirams.re.kr

${ }^{\dagger}$ Corresponding author: Sung Dae Kim. Department of Veterinary Medicine, College of Veterinary Medicine, Kyungpook National University, Daegu 41566, Korea.

Tel: +82-53-950-5958, Fax:+82-53-950-5994, e-mail: kim79sd@knu.ac.kr

(C) The Korean Society for Biomedical Laboratory Sciences. All rights reserved.

(9)This is an Open Access article distributed under the terms of the Creative Commons Attribution Non-Commercial License (http://creativecommons.org/licenses/by-nc/3.0/) which permits unrestricted non-commercial use, distribution, and reproduction in any medium, provided the original work is properly cited. 
an elevation of cytosolic $\mathrm{Ca}^{2+}$ levels by preventing $\mathrm{Ca}^{2+}$ sequestration by the ER in cells (Brüne and Ullrich, 1991). Therefore, it has been widely used to investigate the role of intracellular $\mathrm{Ca}^{2+}$ in various cellular events, including platelet activation (Huang and Kwan, 1998). Thapsig argin induced LDH release in several cell lines such as SK-N-SH neuroblastoma cells, PC12 cells, and SH-SY5Y cells (McGinnis et al., 2001; Janyou et al., 2015). Recent studies explored the use of the TG analog mipsagargin as a therapeutic drug (Mahalingam et al., 2016). However, the use of mipsagargin has been associated with thrombocytopenia as a drug-related adverse event in clinical trials. Nonetheless, few studies have reported the pathophysiological changes in platelets induced by TG. In this study, we investigated the effect of TG on LDH release by platelets. We also investigated whether LDH release could be controlled without affecting platelet aggregation caused by TG.

All reagents including TG (Fig. 1A) were obtained from Sigma-Aldrich (St Louis, MO, USA) unless indicated otherwise. Collagen, thrombin, and ADP were purchased from CHRONO-LOG (Chrono-Log Corp, Havertown, PA, USA). To obtain washed platelets, blood drawn from the vena cavae of Wistar rats was dissolved in 1/5 volume of acid-citrate dextrose (ACD) solution and centrifuged at 1,000 rpm for $7 \mathrm{~min}$. The supernatant containing platelet-rich plasma (PRP) was centrifuged again at $500 \mathrm{rpm}$ for $7 \mathrm{~min}$ to re move red blood cells (RBCs). The PRP was centrifuged at 2,000 rpm for $7 \mathrm{~min}$ and the platelet pellets were resuspended in Tyrode buffer. A complete blood cell (CBC) analyzer (Advia 2120i, Germany) confirmed that the separated platelets were not contaminated with either white blood cells (WBCs) or RBCs (data not shown). The platelet concentration in each experi-
A

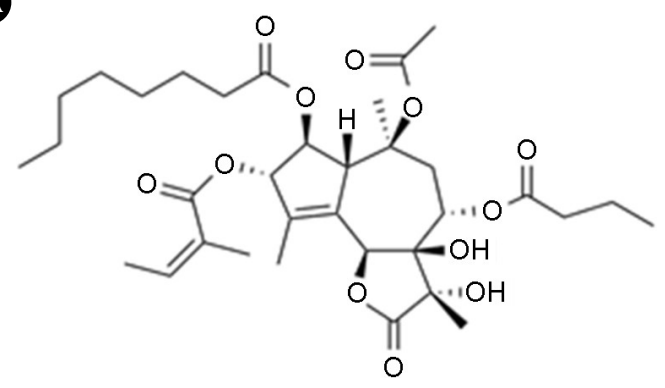

C

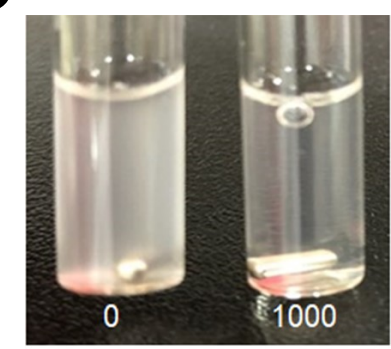

TG (nM)
B



D

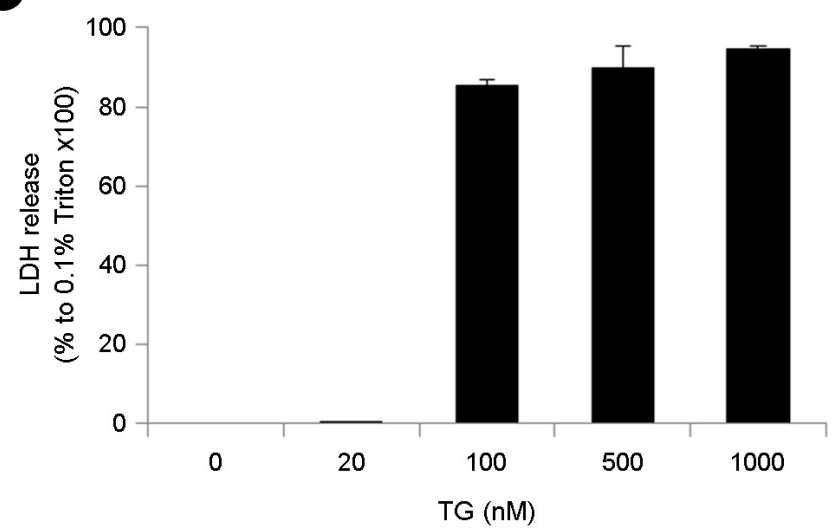

Fig. 1. Effect of thapsigargin (TG) on platelet aggregation and LDH release from platelets. Platelets $\left(4 \times 10^{8} \mathrm{cells} / \mathrm{mL}\right)$ were incubated with TG in the presence of $\mathrm{CaCl}_{2}(1 \mathrm{mM})$ at $37^{\circ} \mathrm{C}$ for $6 \mathrm{~min}$. (A) Chemical structure of TG. (B) TG induced platelet aggregation. (C) Exposure to $1,000 \mathrm{nM}$ TG completely lysed the platelets. (D) $\mathrm{LDH}$ was released from platelets by treatment with 100 to $1,000 \mathrm{nM}$ TG. 
(A)

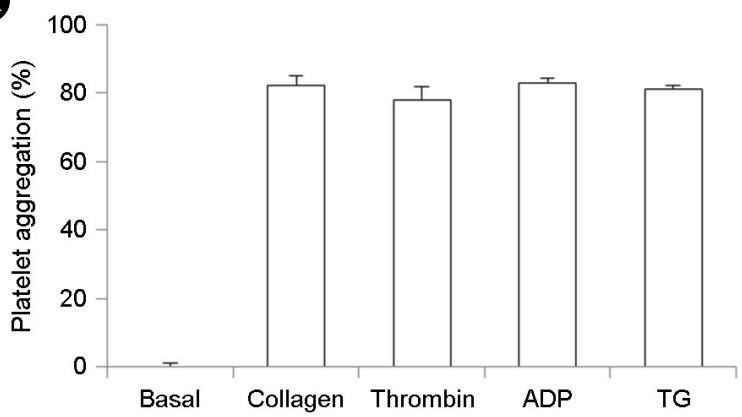

B

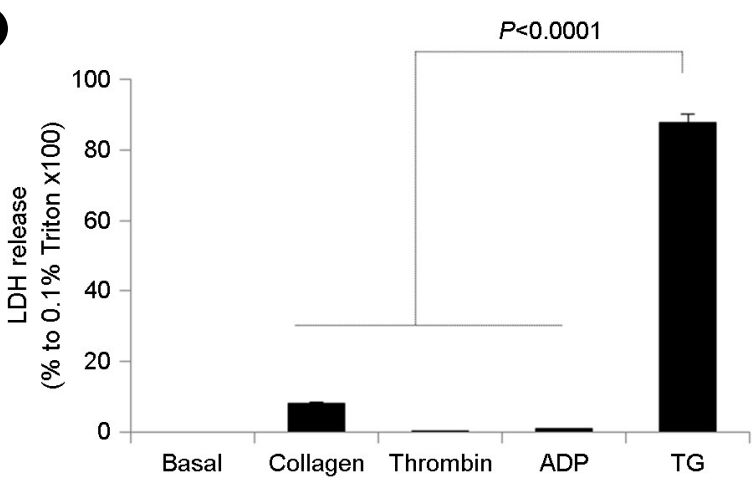

Fig. 2. Comparison of the effect of various agonists on $\mathrm{LDH}$ release from platelets. Platelets $\left(4 \times 10^{8}\right.$ cells $\left./ \mathrm{mL}\right)$ were incubated with various agonists as indicated in the presence of $\mathrm{CaCl}_{2}(1 \mathrm{mM})$ at $37^{\circ} \mathrm{C}$ for 6 min. (A) Platelet aggregation and (B) LDH release were evaluated.

ment was adjusted to $4 \times 10^{8}$ cells $/ \mathrm{mL}$. Ethical approval for this procedure was granted by the Institutional Animal Care and Use Committee of the Dongnam Institute of Radiological and Medical Sciences (DI-2019-008). Platelet aggregation was evaluated photometrically as previously described (Kim et al., 2014). Briefly, the washed platelets (WP) were preincubated with either various pharmacological inhibitors or vehicle for $1 \mathrm{~min}$ at $37^{\circ} \mathrm{C}$ in the presence of $1 \mathrm{mM} \mathrm{CaCl}_{2}$ and then further incubated with thapsigargin for 6 min. After that, the aggregation rate was determined. Thapsigargin was incubated at $37^{\circ} \mathrm{C}$ for $6 \mathrm{~min}$ and then centrifuged at $350 \times \mathrm{g}$ at room temperature for $3 \mathrm{~min}$. A $100 \mu \mathrm{L}$ aliquot of the supernatant was used to measure $\mathrm{LDH}$ leakage from platelets and quantified using the FUJI DRYCHEM 4000i (Fuji, Tokyo, Japan). The total amount of released LDH was calculated as a percentage of the total LDH leakage measured in washed platelets completely lysed with $0.1 \%$ Triton $\mathrm{X}-100$. The results are presented as the mean \pm standard error of the mean (SEM). We considered a $P$-value of less than 0.05 as statistically significant. Statistical analysis was performed using SPSS ver. 18.0.

To determine the toxic effect of TG on platelets, we first performed a light transmission assay. As shown in Fig. 1B, the platelets were aggregated up to $85 \%$ at $100 \mathrm{nM}$ TG Surprisingly, we also found that $1,000 \mathrm{nM}$ TG treatment completely lysed the platelets (Fig. 1C). LDH is a cytoplasmic enzyme rapidly released by damaged cells. Hence, we evaluated the LDH release from platelets treated with TG. We observed that platelet LDH release was also increased by TG treatment, similar to the platelet aggregation response to TG (Fig. 1D). To determine whether LDH release following TG treatment was a TG-dependent effect, LDH release from treatment with other agonists such as collagen $(2.5 \mu \mathrm{g} / \mathrm{mL})$, thrombin $(0.1 \mathrm{U} / \mathrm{mL})$, and ADP $(10 \mu \mathrm{M})$ was also investigated. As shown in Fig. 2A, all of the agonists induced platelet aggregation, which means that our experimental system was normal. However, LDH release was only induced by TG, and no significant level of LDH release was observed by treatment with other agonists (Fig. 2B). These results indicated that the platelet aggregation reaction caused by agonists was not necessarily accompanied by the release of LDH. To investigate the correlation between the TGinduced platelet aggregation reaction and LDH release, we analyzed TG-induced light transmission and platelet LDH levels in a time-dependent manner. As shown in Fig. 3A, platelet aggregation started within 1 minute after $100 \mathrm{nM}$ TG treatment, followed by the release of $\mathrm{LDH}$ at the same time or shortly thereafter. Fig. 3B shows the corresponding images of platelets after TG treatment. These results showed a close correlation between TG-induced platelet aggregation and LDH release, and TG-induced platelet aggregation reactions appeared to precede LDH release. Since MAPKs are involved in the platelet activation process, we next assessed whether MAPKs participated in the regulation of TGinduced platelet activation and LDH release using MAPKspecific inhibitors such as PD90859 (an ERK 1/2 inhibitor), SB203580 (a p38 MAPK inhibitor), and SP600125 (a JNK inhibitor). As shown in Fig. 4, none of the three inhibitors inhibited TG-induced platelet aggregation. Only SP600125 inhibited TG-induced LDH release. PI3K-Akt pathways 


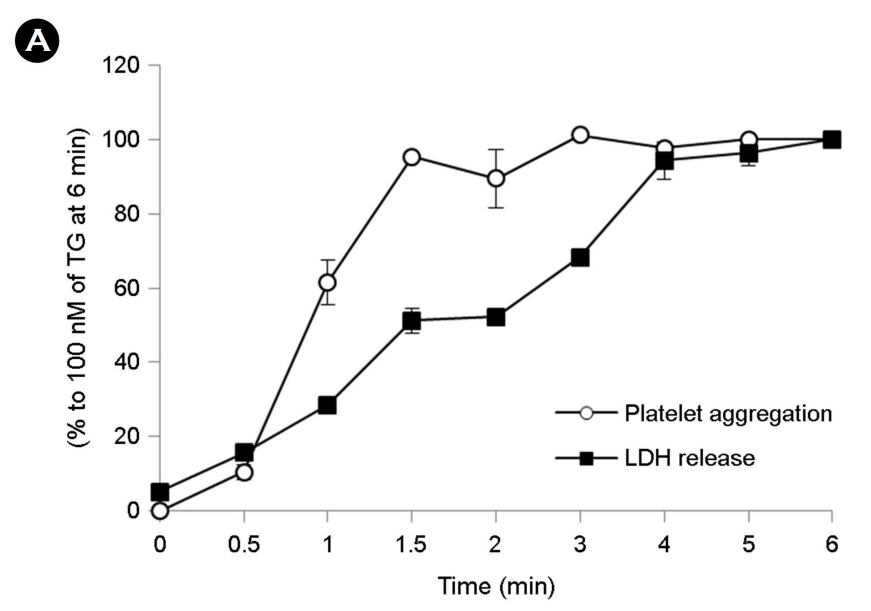

Fig. 3. (A) Temporal variation in platelet aggregation and LDH release following $100 \mathrm{nM}$ TG treatment. (B) Corresponding microscopic images of TG-treated platelets over time. Scale bar $=400 \mu \mathrm{m}$.

B

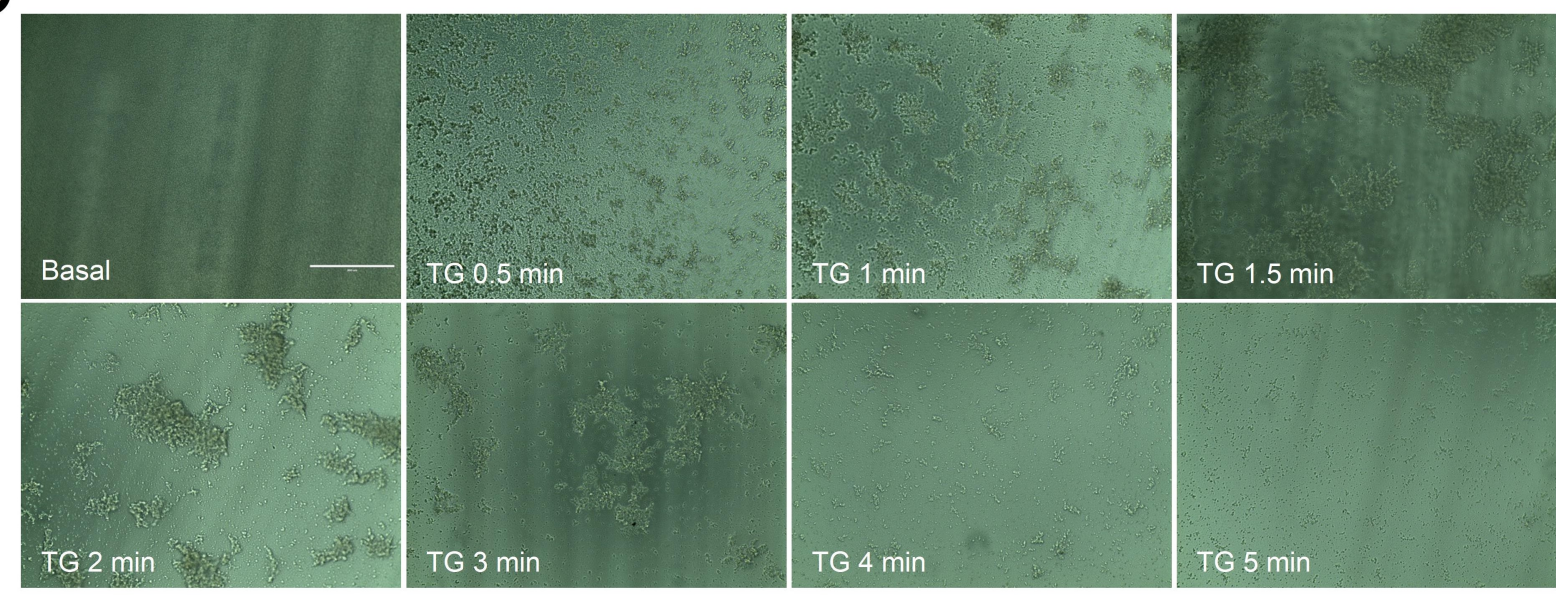

A

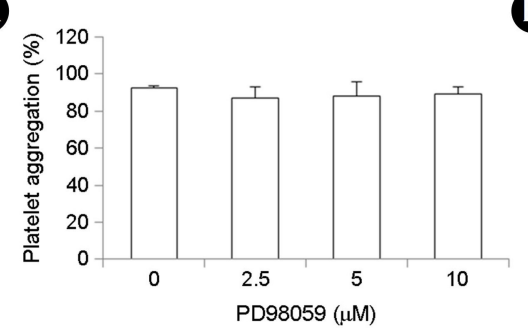

D

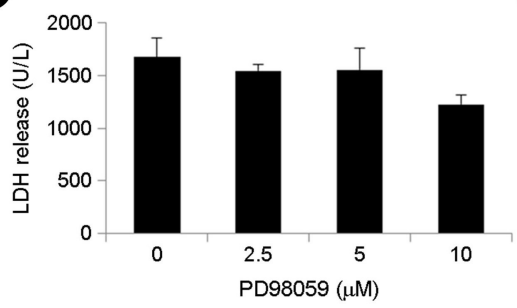

B

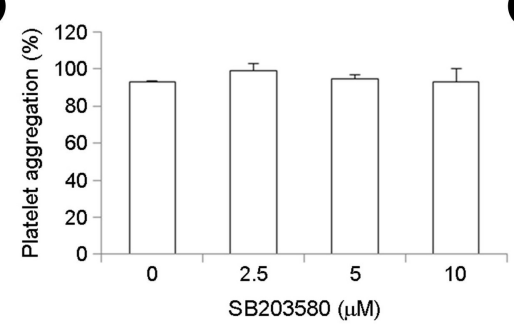

E

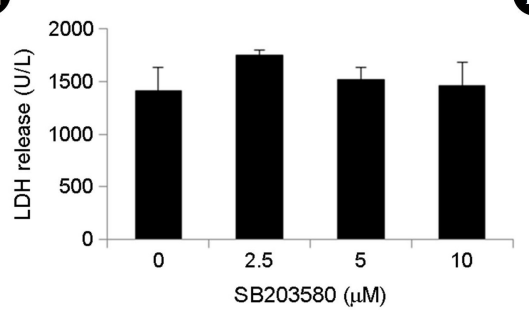

C

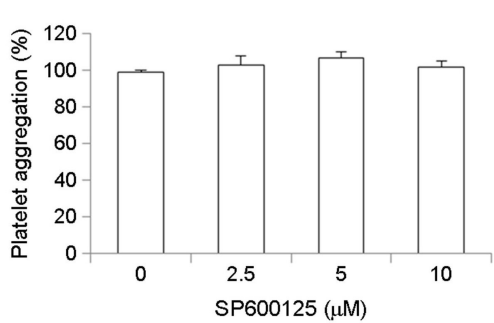

$\boldsymbol{F}$

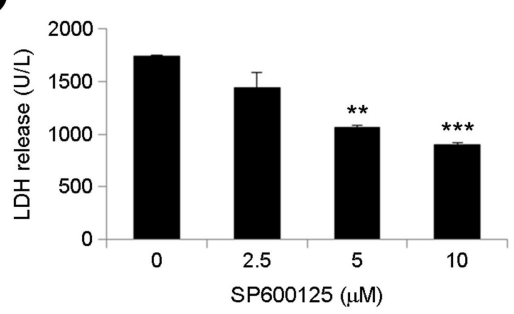

Fig. 4. Effect of MAPK inhibitors on TG-induced platelet aggregation and LDH release from platelets. Platelets $\left(4 \times 10^{8}\right.$ cells $\left./ \mathrm{mL}\right) \mathrm{were}$ incubated with or without PD98059 (A and D), SB203580 (B and E), and SP600125 (C and F) at $37^{\circ} \mathrm{C}$ for 1 min with stirring, followed by the addition of TG. Additional incubation was performed for $6 \mathrm{~min}$. The cells were then centrifuged to measure the LDH released into the supernatant. Platelet aggregation (A, B, and C) and LDH release (D, E, and F) are shown as bar graphs. $* * P<0.01, * * * P<0.001$ versus 100 nM TG-treated platelets. 
A

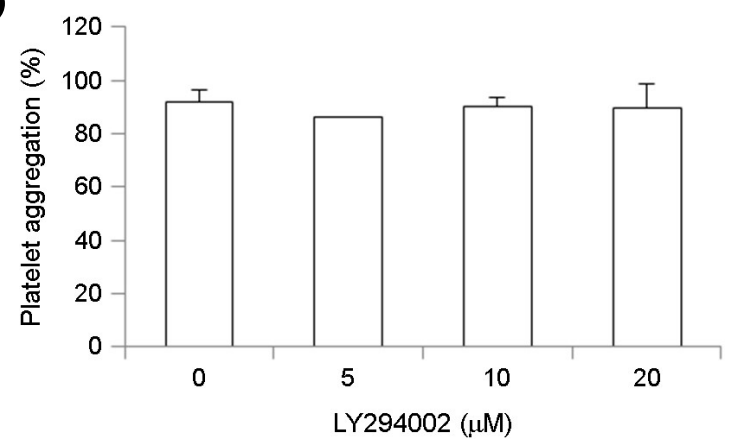

B

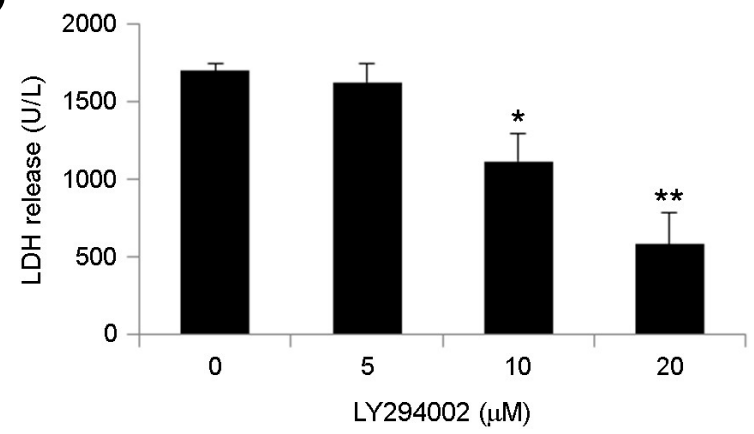

Fig. 5. Effect of PI-3K inhibitors on TG-induced platelet aggregation and LDH release from platelets. Platelets $\left(4 \times 10^{8}\right.$ cells $\left./ \mathrm{mL}\right)$ were incubated with or without LY294002 at $37^{\circ} \mathrm{C}$ for $1 \mathrm{~min}$ with stirring, followed by the addition of TG. Additional incubation was performed for $6 \mathrm{~min}$. The cells were then centrifuged to measure the LDH released into the supernatant. Platelet aggregation (A) and LDH release (B) are shown as bar graphs. $* P<0.05, * * P<$ 0.01 versus $100 \mathrm{nM}$ TG-treated platelets.

are pivotal regulator axes governing platelet activation and aggregation. Hence, we also assessed whether PI3K-Akt pathways participated in regulating TG-induced platelet activation and LDH release using the PI3K specific inhibitor LY294002. As shown in Fig. 5, LY294002 did not inhibit TG-induced platelet aggregation and LDH release. Elevated intracellular cAMP concentrations have been reported to promote platelet stabilization in the presence of agonistinitiated platelet aggregation. Hence, we also assessed whether the cAMP production participated in regulating TG-induced platelet activation and LDH release using the adenylyl cyclase activator forskolin. As shown in Fig. 6, forskolin did not inhibit TG-induced platelet aggregation and LDH release.

TG, an inhibitor of the sarco/endoplasmic reticulum (ER) $\mathrm{Ca}^{2+}$-ATPase (SERCA), has been widely used as an agonist
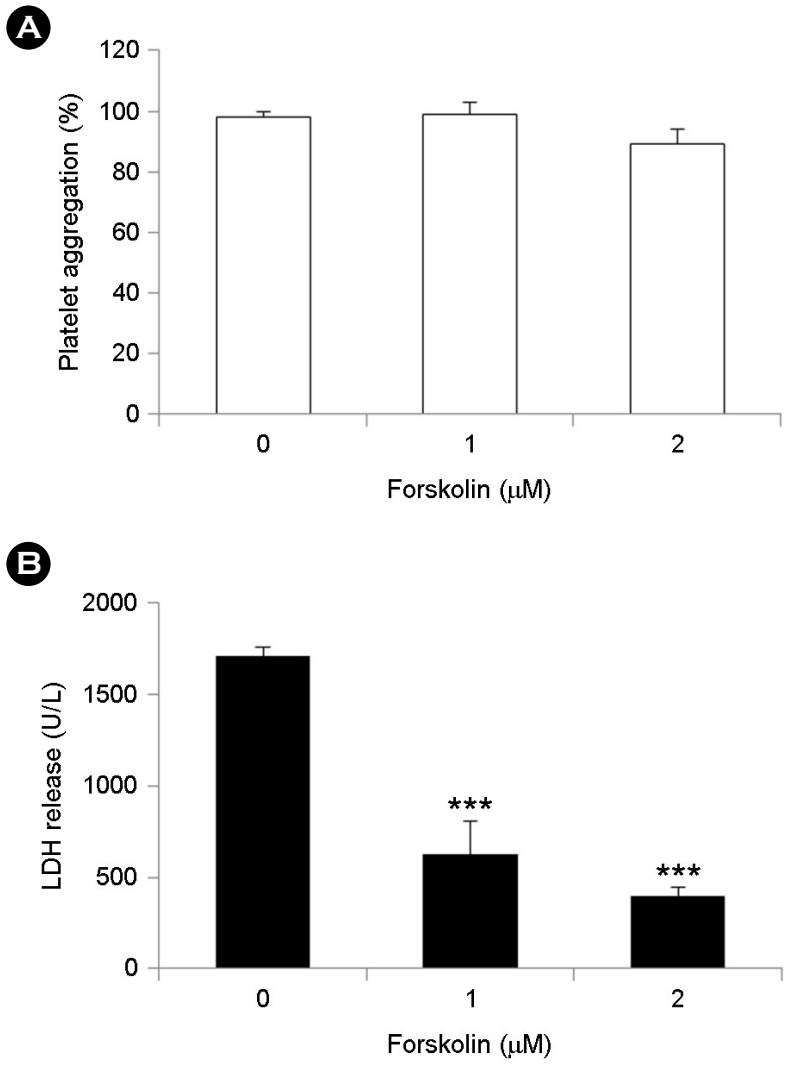

Fig. 6. Effect of cAMP stimulator on TG-induced platelet aggregation and LDH release from platelets. Platelets $\left(4 \times 10^{8}\right.$ cells $\left./ \mathrm{mL}\right)$ were incubated with or without forskolin at $37^{\circ} \mathrm{C}$ for $1 \mathrm{~min}$ with stirring, followed by the addition of TG. Additional incubation was performed for $6 \mathrm{~min}$. The cells were then centrifuged to measure the $\mathrm{LDH}$ released into the supernatant. Platelet aggregation (A) and LDH release (B) are shown as bar graphs. $* * * P<0.001$ versus $100 \mathrm{nM}$ TG-treated platelets.

of platelet aggregation since the early 1990s (Brüne and Ullrich, 1991). Although there have been various reports related to the increase in intracellular $\mathrm{Ca}^{2+}$ influx in platelets treated with TG and the subsequent platelet aggregation, there have been no reports on platelet lysis and LDH release by TG treatment. Here, we showed the complete lysis of and $\mathrm{LDH}$ release from platelets treated with TG for the first time. In this regard, a recent report also suggested the role of the thapsigargin-based prostate-specific membrane antigen (PSMA)-activated prodrug mipsagargin as an anti-cancer drug, and clinical trials reported thrombocytopenia as a drugrelated adverse event (Mahalingam et al., 2016). As broadspectrum protein kinase $\mathrm{C}(\mathrm{PKC})$ inhibitors reduce secretion and aggregation, the PKC family is generally considered a 
positive platelet activation regulator (Harper and Poole, 2010). In our experiments, Ro 31-8220, a PKC inhibitor, also strongly inhibited TG-induced platelet aggregation and LDH release (data not shown). Until this stage, TG-induced platelet aggregation and LDH release were thought to substantially share a common signaling pathway. The results suggest that LDH is released only after platelet aggregation induced by TG treatment (Fig. 3), implying that only the pathological response (LDH release) of platelets to TG was targeted and controlled, without affecting the physiological response (platelet aggregation). To control the LDH release from platelets without affecting platelet aggregation by TG, agents that were reported to inhibit platelet aggregation were used. In this study, we found three pharmacological inhibitors that specifically controlled the LDH release induced by TG in platelets: SP600125 (a JNK MAPK inhibitor), LY294002 (a PI-3K inhibitor), and forskolin (an adenylyl cyclase activator). Several studies reported the role of MAPK in agonist-induced platelet aggregation via integrin activation and thrombus formation. Three subgroups of MAP kinases have been defined, extracellular signal-regulated kinases (ERKs), p38, and c-Jun NH2-terminal kinases (JNKs). JNKs have been implicated in the apoptosis of diverse cells (Engedal et al., 2002; Wu et al., 2019) but little is known about either the activation or role of JNK in platelets compared to the other two ERKs and p38 MAPK (Adam et al., 2008). In this study, we showed that the role of JNK in TGinduced platelet activation was mediated by the inhibition of LDH release by TG without affecting platelet aggregation. The other two MAPK inhibitors did not inhibit platelet LDH release by TG treatment. Phosphoinositide 3-kinases (PI3Ks), also called phosphatidylinositol 3-kinases, are involved in cellular functions including platelet activation. In our study, the pan-PI3K inhibitor LY294002 inhibited TG-induced LDH release from platelets. LY294002 (5 to $20 \mu \mathrm{M}$ ) had no inhibitory effect on the TG-induced platelet aggregation. In a previous report, the pan-PI3K inhibitor LY294002 (20 $\mu \mathrm{M})$ inhibited collagen-induced platelet aggregation. In addition, the inhibition of PI 3-kinase by wortmannin $(20 \mathrm{nM})$ or LY294002 $(20 \mu \mathrm{M})$ abolished platelet secretion and aggregation, as well as phospholipase C (PLC) activation (Yi et al., 2014). This was thought to be due to the difference in the mechanisms by which the agonists (collagen versus thapsigargin) used acted on platelets, and an in-depth study on this is needed in the future. Elevated intracellular cAMP concentrations have been reported to promote platelet stabilization in the presence of agonist-initiated platelet aggregation. By activating the protein kinases that phosphorylate the $\mathrm{Ca}^{2+}$ pump, $\mathrm{Ca}^{2+}$ uptake into the dense tubular system is decreased, resulting in the inhibition of platelet activation cascades (Collazos and Sanchez, 1987; Liu et al., 2009). Forskolin, a diterpene obtained from coleus forskolin, directly stimulates adenylyl cyclase to increase cAMP levels in platelets. Forskolin also had a vaso-relaxing effect in an in vivo experimental model (Christenson et al., 1995). Furthermore, forskolin reduced whole blood platelet aggregation and ATP release induced by collagen and ADP in a dose-dependent fashion (Kariya et al., 1985). Therefore, it was expected to prevent TG-induced platelet aggregation. In this study, we found that forskolin inhibited TG-induced LDH release from platelets without inhibiting platelet aggregation. This was thought to be because TG directly increased $\mathrm{Ca}^{2+}$ influx in the cytoplasm through $\mathrm{Ca}^{2+}$ store-activated $\mathrm{Ca}^{2+}$-channels without binding platelet receptors (Huang and Kwan, 1998). This means that only LDH release was controlled, without affecting platelet aggregation by TG. Advances in the treatment and prognosis of disease based on the correlation between platelets and serum LDH levels suggest an association between elevated LDH levels and thrombocytopenia. Patton et al. reported that serum LDH and platelet counts were predictive biomarkers of survival in patients with thrombotic thrombocytopenic purpura (Patton et al., 1994). A negative correlation between serum LDH levels and platelet counts in patients with immune thrombocytopenia was also reported (Al-Samkari and Kuter, 2019).

In conclusion, the results of this study provide evidence that 1) TG induced both platelet aggregation and $\mathrm{LDH}$ release, 2) LDH release by TG was not an event independent of TG-induced platelet aggregation but a sequential event, and 3) only LDH release was precisely controlled, without affecting the platelet aggregation reaction by TG. Although the exact mechanism of $\mathrm{LDH}$ release caused by TG remains elusive, our data suggest a role for platelets in response to pathophysiological stress that causes platelet death or damage 
such as thrombocytopenia.

\section{ACKNOWLEDGEMENT}

This work was supported by a National Research Foundation of Korea (DIRAMS) grant funded by the Korean government (MSIP) (50591-2021).

\section{CONFLICT OF INTEREST}

The authors declare that they have no conflict of interest.

\section{REFERENCES}

Adam F, Kauskot A, Rosa JP, Bryckaert M. Mitogen-activated protein kinases in hemostasis and thrombosis. Journal of Thrombosis and Haemostasis. 2008. 6: 2007-2016.

Al-Samkari H, Kuter DJ. Lactate dehydrogenase is elevated in immune thrombocytopenia and inversely correlates with platelet count. British Journal of Haematology. 2019. 187: e61-e64.

Brüne B, Ullrich V. Calcium mobilization in human platelets by receptor agonists and calcium-ATPase inhibitors. FEBS Letters. 1991. 284: 1-4.

Christenson JT, Thulesius O, Nazzal MM. The effect of forskolin on blood flow, platelet metabolism, aggregation and ATP release. Vasa. 1995. 24: 56-61.

Collazos JM, Sanchez A. cAMP reduces the affinity of $\mathrm{Ca}^{2+}$ triggered secretion in platelets. FEBS Letters. 1987. 215: 183186.

Engedal N, Korkmaz CG, Saatcioglu F. C-Jun N-terminal kinase is required for phorbol ester- and thapsigargin-induced apoptosis in the androgen responsive prostate cancer cell line LNCaP. Oncogene. 2002. 21: 1017-1027.

Harper MT, Poole AW. Diverse functions of protein kinase C isoforms in platelet activation and thrombus formation. Journal of Thrombosis and Haemostasis. 2010. 8: 454-462.

Huang SJ, Kwan CY. Cyclopiazonic acid and thapsigargin induce platelet aggregation resulting from $\mathrm{Ca}^{2+}$ influx through $\mathrm{Ca}^{2+}$ store-activated $\mathrm{Ca}^{2+}$-channels. European Journal of Pharmacology. 1998. 341: 343-347.

Janyou A, Changtam C, Suksamrarn A, Tocharus C, Tocharus J. Suppression effects of O-demethyldemethoxycurcumin on thapsigargin triggered on endoplasmic reticulum stress in SK-N-SH cells. Neurotoxicology. 2015. 50: 92-100.

Kariya T, Morito F, Sakai T, Takahata K, Yamanaka M. Effect of forskolin on platelet deaggregation and cyclic AMP generation. Naunyn-Schmiedeberg's Archives of Pharmacology. 1985. 331: 119-121.

Kim SD, Lee YJ, Baik JS, Han JY, Lee CG, Heo K, Park YS, Kim JS, Ji HD, Park SI, Rhee MH, Yang K. Baicalein inhibits agonist- and tumor cell-induced platelet aggregation while suppressing pulmonary tumor metastasis via cAMP-mediated VASP phosphorylation along with impaired MAPKs and PI3K-Akt activation. Biochemical Pharmacology. 2014. 92: 251-265.

Liu FC, Liao CH, Chang YW, Liou JT, Day YJ. A new insight of anti-platelet effects of sirtinol in platelets aggregation via cyclic AMP phosphodiesterase. Biochemical Pharmacology. 2009. 77: 1364-1373.

Mahalingam D, Wilding G, Denmeade S, Sarantopoulas J, Cosgrove D, Cetnar J, Azad N, Bruce J, Kurman M, Allgood VE, Carducci M, Mipsagargin, a novel thapsigargin-based PSMAactivated prodrug: results of a first-in-man phase I clinical trial in patients with refractory, advanced or metastatic solid tumours. British Journal of Cancer. 2016. 114: 986-994.

McGinnis KM, Wang KK, Gnegy MM. Calcium/calmodulindependent protein kinase inhibition potentiates thapsigarginmediated cell death in SH-SY5Y human neuroblastoma cells. Neuroscience Letters. 2001. 301: 99-102.

Patton JF, Manning KR, Case D Owen, J. Serum lactate dehydrogenase and platelet count predict survival in thrombotic thrombocytopenic purpura. American Journal of Hematology. 1994. 47: 94-99.

Wu L, Huang X, Kuang Y, Xing Z, Deng X, Luo Z. Thapsigargin induces apoptosis in adrenocortical carcinoma by activating endoplasmic reticulum stress and the JNK signaling pathway: an in vitro and in vivo study. Drug Design, Development and Therapy. 2019. 13: 2787-2798

Yi W, Li Q, Shen J, Ren L, Liu X, Wang Q, He S, Wu Q, Hu H, Mao X, and Zhu L. Modulation of Platelet Activation and Thrombus Formation Using a Pan-PI3K Inhibitor S14161. PLoS One. 2014. 9: e102394.

https://doi.org/10.15616/BSL.2021.27.3.170

Cite this article as: Baik JS, Seo YN, Rhee MH, Park MT, Kim SD. Thapsigargin Induces Platelet Aggregation, thereby Releases Lactate Dehydrogenase from Rat Platelets. Biomedical Science Letters. 2021. 27: 170-176. 